\title{
PERSPECTIVES OF POLARONS
}




\section{Published by}

World Scientific Publishing Co. Pte. Ltd.

P O Box 128, Farrer Road, Singapore 912805

USA office: Suite 1B, 1060 Main Street, River Edge, NJ 07661

UK office: 57 Sheiton Street, Covent Garden, London WC2H 9HE

\section{Library of Congress Cataloging-in-Publication Data}

Perspectives of polarons / editors G. N. Chuev, V. D. Lakhno.

p. $\mathrm{cm}$.

ISBN 9810227787

1. Polarons. I. Chuev, G. N. (Gennady N.), 1961-

II. Lakhno, V. D. (Viktor D.)

QC176.8.P62P47 1996

$530.4^{\prime} 16--\mathrm{dc} 20$

96-35326

CIP

\section{British Library Cataloguing-in-Publication Data}

A catalogue record for this book is available from the British Library.

Copyright @ 1996 by World Scientific Publishing Co. Pte. Ltd.

All rights reserved. This book, or parts thereof, may not be reproduced in any form or by any means, electronic or mechanical, including phorocopying, recording or any information storage and retrieval system now known or to be invented, without written permission from the Publisher.

For photocopying of material in this volume, please pay a copying fee through the Copyright Clearance Center, Inc., 222 Rosewood Drive, Danvers, MA 01923, USA. In this case permission to photocopy is not required from the publisher.

This book is printed on acid-free paper.

Printed in Singapore by Uto-Print 
The monograph "Perspectives of polarons" presents further development of the polaron theory and its applications in various fields of physics. Originally polaron arose in condensed media physics to explain peculiarities of electron states in semiconductors, ionic crystals and other polar media. Subsequently methods of the polaron theory were used to describe electron properties in other condensed media. The states of polaron type in nonpolar media were named as condenson, in magnetoordered media as magnet polaron, in the electrolytes as ionon, etc. Nowadays methods of the polaron theory are widely used in such sciences as biology, chemistry, nuclear physics, etc. Such wide applications result from the fact that polaron is one of the first substantial examples of a nonlinear quasiparticle.

This monograph deals with only a small part of the problems indicated. It is based on the talks, given at the Workshops which were held in Pushchino in 1993 and 1994.

Our book includes selected articles, which are representative of the exciting scientific presentations that marked the workshops. The first Chapter is devoted to theory and applications of large polaron. The combination of polaron theory with modern quantum chemical calculations is presented in Chapter 2. The polaron problem includes issues which are being studied intensively in the quantum field theory and nonperturbative models of elementary particles. We include three works (Chapters 3, 4, and 13) devoted to applications of the polaron in quantum field theory of particles. The polaron behavior in magnetic systems and magnetic fields are considered in Chapters 6, 10, and 12. Effects of disorder and order of the structure on self-trapped electron states are treated in Chapters 7 and 8. We include the review devoted to quasi-one-dimensional polaron (Chapter 5) in biopolymers, due to vast number of applications of polaron model in one-dimensional structures. The study of high-temperature superconductivity stimulates the bipolaron theory. A generalization of the bipolaron problem to the anisotropic case is considered in Chapter 9.

We hope our book will be of interest to a broad range of readers, which can be able to sense the excitment of the articles.

The monograph will hopely surve further development and contribute to the introduction of the polaron theory methods into various fields of physics.

\author{
Gennady N.Chuev
}

\author{
Victor D.Lakhno
}


This page is intentionally left blank 


\section{CONTENTS}

PART 1. Papers based on the workshop "Perspectives in Polarons" (Pushchino, 1993).

Theory and Applications of Strongly Coupled Large Polaron

G. N. Chuev and V. D. Lakhno

Theoretical Investigation of Self-Trapped Hole in Alkali Halides.

Long-Range Effects Within the Model Hamiltonian Approach

P. B. Zapol and L. N. Kantorovich

The Quantumfield Theory of Binucleon

V. D. Lakhno

Generalized Functional Approach in the Theory Meson-Nucleon

Interaction

V. D. Lakhno

Nonlinear Mathematical Models in Biopolymer Science

L. V. Yakushevich

The Ground State of Fröhlich 1D Fermi-Polaron

E. A. Kochetov

PART 2. Papers based on the workshop "Autolocalized Electron States in Ordered and Disordered Systems" (Pushchino, 1994).

Mean-Field Theory of the Solvated Electron and Dielectron States

G. N. Chuev

Resonance, Localized and Polaron-Type Electron States in

Elastic Materials with Topological Defects

V. A. Osipov

Bipolarons in Anisotropic Crystals

N. I. Kashirina, E. V. Mozdor, E. A. Pashitskij, and V. I. Sheka

SU(2) Path Integral for the Heisenberg Ferromagnetic

E. A. Kochetov 
Low-Temperature Electron Mobility of Acoustical Polaron

B. A. Kotiya and V. F. Los

Polaron Effects on Magnitoresistance in Quasi-Two-Dimensional Conductors

L. S. Kukushkin

Numerical Investigation of a Quantumfield Model for Strong-Coupled Binucleon

I. V. Amirkhanov, I. V. Puzynin, T. P. Puzynina, T. A. Strizh, E. V. Zemlyanaya and V. D. Lakhno 


\section{AUTHOR's ADDRESSES}

Dr. I.V. Amirkhanov

Laboratory of Computing Techniques and Automation Joint Institute for Nuclear Research, Dubna, 141980 Russia

Prof. G.N. Chuev Department of Quantum-Mechanical Systems, Institute of Mathematical Problem of Biology of Russian Academy of Sciences, Pushchino, 142292 Russia

Dr. L.N. Kantorovich

Department of Physics, University of Keele, Keele, Staffordshire, ST5 5BG,UK

Dr. N.I.Kashirina

Institute of Physics of Semiconductors of National Ukraine Academy of Science, Kiev 25202, Ukraine

Dr.E.A.Kochetov

Laboratory of Theoretical Physics, Joint Institute for Nuclear Research Dubna 141980, Russia

Prof. B.A.Kotiya

Georgian Technical University, Tbilisi, Georgia

Prof. L.S.Kukushkin Physical and Technical Institute of Low Temperature, Kharkov, Ukraine 
Prof. V.D. Lakhno

Department of Quantum-Mechanical Systems, Institute of Mathematical Problem of Biology of Russian Academy of Sciences, Pushchino, 142292 Russia

Prof. V.F.Los

Institute of Metal Physics, Kiev, Ukraine

Dr. E.V, Mozdor

Institute of Physics of Semiconductors of National Ukraine Academy of Science, Kiev 25202, Ukraine

Dr. V.A.Osipov

Laboratory of Theoretical Physics, Joint Institute for Nuclear Research

Dubna 141980, Russia

Prof. E.A.Pashitskij

Institute of Physics of Semiconductors of National Ukraine Academy of Science, Kiev 25202, Ukraine

Prof. I.V.Puzynin

Laboratory of Computing Techniques and Automation Joint Institute for Nuclear Research, Dubna, 141980 Russia

Dr. T.P. Puzynina, Laboratory of Computing Techniques and Automation Joint Institute for Nuclear Research, Dubna, 141980 Russia

Prof. V.I. Sheka

Institute of Physics of Semiconductors

of National Ukraine Academy of Science, Kiev 25202, Ukraine 
Dr. T.A.Strizh

Laboratory of Computing Techniques and Automation Joint Institute for Nuclear Research, Dubna, 141980 Russia

Dr. L.V. Yakushevich

Institute of Cell Biophysics, Russian Academy of Sciences, 142292 Pushchino, Moscow Region, Russia

Dr. E.V. Zemlyanaya

Laboratory of Computing Techniques and Automation Joint Institute for Nuclear Research, Dubna, 141980 Russia

Dr. P.Zapol

Department of Physics,

Michigan Technological University, Houghton, MI, 49931, USA 